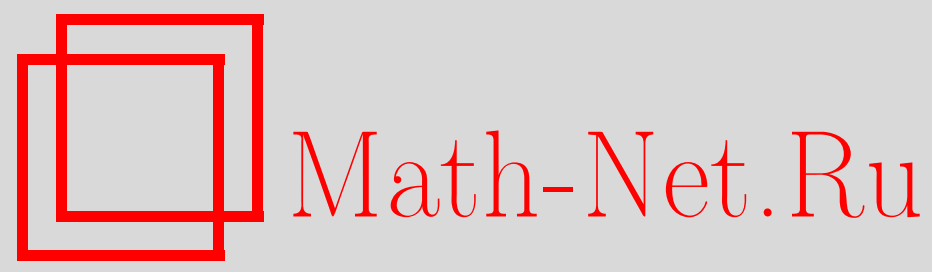

А. А. Боровков, Об асимптотике распределений времен первого прохождения II, Матем. заметки, 2004, том 75, выпуск 3, 350-359

DOI: https://doi.org/10.4213/mzm37

Использование Общероссийского математического портала Math-Net.Ru подразумевает, что вы прочитали и согласны с пользовательским соглашением http://www.mathnet.ru/rus/agreement

Параметры загрузки:

IP: 34.239 .49 .27

26 апреля 2023 г., $17: 39: 19$ 
УДК 519.214

\section{ОБ АСИМПТОТИКЕ РАСПРЕДЕЛЕНИЙ ВРЕМЕН ПЕРВОГО ПРОХОЖДЕНИЯ ІІ}

\section{А. А. Боровков}

В работе найдена асимптотика и получены оценки для распределения времен первого прохождения нулевого уровня (в обоих направлениях) случайным блужданием с ненулевым сносом.

Библиографоия: 22 названия.

\section{1. Введение}

Настоящая работа является продолжением [1]. Пусть $\xi, \xi_{1}, \xi_{2}, \ldots$ - независимые одинаково распределенные случайные величины, $S_{n}=\sum_{j=1}^{n} \xi_{j}$,

$$
\eta_{+}(x)=\min \left\{k: S_{k}>x\right\}, \quad \eta_{-}(x)=\min \left\{k \geqslant 1: S_{k} \leqslant-x\right\},
$$

$x \geqslant 0, \eta_{ \pm}(0)=\eta_{ \pm}$.

$\mathrm{B}[1]$ в $\oint 1$ была изучена асимптотика $\mathbf{P}\left(\eta_{-}(x)>n\right)$ и $\mathbf{P}\left(\eta_{+}(x)=n\right)$ в случае $x=0$ и отрицательного сноса, когда вьполнено условие

$$
A_{-}=\left\{D_{+}=\sum \frac{\mathbf{P}\left(S_{k}>0\right)}{k}<\infty, \quad D_{-}=\sum \frac{\mathbf{P}\left(S_{k} \leqslant 0\right)}{k}=\infty\right\}
$$

При этом были рассмотрены следующие три класса распределений $\xi$ :

$\mathscr{R}$ - класс регулярных распределений, когда $\mathbf{P}(\xi>x)=V(x)$, где

$$
V(x)=x^{-\alpha} L(x), \quad \alpha>1
$$

$L(x)$ - медленно меняющаяся функция (ММФ);

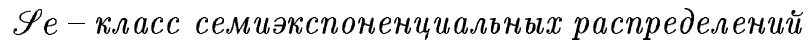

$$
\mathbf{P}(\xi>x)=V(x)=e^{-l(x)}, \quad l(x)=x^{\alpha} L(x), \quad \alpha \in(0,1),
$$

Работа выполнена при частичной финансовой поддержке Российского фонда фундаментальных исследований, гранты №№ 02-01-00902, 00-15-96178, и INTAS, проект № 00-265. 
где $L-\mathrm{MM} \Phi$ такая, что при $\Delta=o(x), x \rightarrow \infty$ и любом $\varepsilon>0$

$$
\begin{array}{ll}
l(x+\Delta)-l(x) \sim \frac{\alpha \Delta l(x)}{x}, & \text { если } \frac{\alpha \Delta l(x)}{x}>\varepsilon, \\
l(x+\Delta)-l(x) \rightarrow 0, & \text { если } \frac{\alpha \Delta l(x)}{x} \rightarrow 0 ;
\end{array}
$$

$\mathscr{C}$ - альтернативный $\kappa \mathscr{R}$ и $\mathscr{S}$ класс распределений, убывающих әкспоненциально быстро, т.е. распределений, для которых выполнено условие Крамера:

$$
\mu=: \sup \{\lambda: \varphi(\lambda)<\infty\}>0, \quad \text { где } \varphi(\lambda)=: \mathrm{E} e^{\lambda \xi} .
$$

Перейдем теперь к рассмотрению случаев, когда $x \geqslant 0$ фиксировано и когда $x \rightarrow \infty$.

\section{2. Случай, когда $x \geqslant 0$ фиксировано}

2.1. Случай $A_{0}=\left\{D_{+}=\infty, D_{-}=\infty\right\}$. Отметим прежде всего, что здесь имеет место следующий аналог теоремы 1.

ТЕорема 7. При выполнении $A_{0}$

$$
\frac{1-\mathrm{E} z^{\eta_{-}}}{1-z}=\exp \left\{\sum_{k=1}^{\infty} \frac{z^{k}}{k} \mathbf{P}\left(S_{k}>0\right)\right\}=\frac{1}{1-\mathrm{E} z^{\eta_{+}}},
$$

$\mathrm{E} \eta_{ \pm}=\infty$, распределение $\eta_{-}$однозначно определяется распределением $\eta_{+}$и наобopom.

ДоКАЗАТЕЛЬСТво теоремы очевидным образом следует из (18), (19) в [1] и того, что $\mathbf{P}\left(\eta_{+}<\infty\right)=1$.

Далее имеет место следующее утверждение. Обозначим

$$
\Delta_{n}=\mathbf{P}\left(S_{n} \leqslant 0\right)-\gamma, \quad G(z)=\exp \left\{-\sum_{k=1}^{\infty} \frac{z^{n} \Delta_{n}}{n}\right\} .
$$

Teopema 8. B cлучаe $A_{0}$

1)

$$
\mathbf{P}\left(\eta_{-}>n\right) \sim \frac{n^{-\gamma} L(n)}{\Gamma(1-\gamma)}, \quad \gamma \in(0,1),
$$

при $n \rightarrow \infty$, где $L-M M \Phi$, тогда и только тогда, когда

$$
n^{-1} \sum_{k=1}^{n} \mathbf{P}\left(S_{k} \leqslant 0\right) \rightarrow \gamma
$$

2) при выполнении (4) или (5) с необходимостью $L(n) \sim G(1-1 / n)$,

$$
\frac{\mathbf{P}\left(\eta_{-}(x)>n\right)}{\mathbf{P}\left(\eta_{-}>n\right)} \rightarrow r_{-}(x)
$$

при $n \rightarrow \infty u$ любом фиксированном $x \geqslant 0$, где функиия $r_{-}(x)$ найдена в явном виде;

3) если $\mathrm{E} \xi=0, \mathrm{E} \xi^{2}<\infty$, mo $\gamma=1 / 2 u$

$$
\sum \frac{\left|\Delta_{n}\right|}{n}<\infty
$$

Это означает, что $L(n)$ в (4) мохсно заменить на $G(1), 0<G(1)<\infty$. 
ДокАЗАТЕЛЬСТво теоремы 8 см. в [2, с. 381, 382] (там же см. подробную библиографию). Очевидно, что симметричное к (4) утверждение справедливо для $\eta_{+}, \eta_{+}(x)$ (c заменой $\gamma$ и $\Delta_{n}$ соответственно на $1-\gamma$ и $\left.-\Delta_{n}\right)$ :

$$
\mathbf{P}\left(\eta_{+}>n\right) \sim \frac{n^{\gamma-1} L^{-1}(n)}{\Gamma(\gamma)} .
$$

Если $\mathrm{E} \xi^{2}=\infty$ (если $\mathrm{E} \xi$ существует, то $\left.\mathrm{E} \xi=0\right)$, то при выполнении известных условий на регулярность хвостов распределения $\xi$ вьполняется

$$
\mathbf{P}\left(S_{n} \leqslant 0\right) \rightarrow F_{\alpha, \theta}(0) \equiv \gamma,
$$

где $F_{\alpha, \theta}$ - функция распределения устойчивого закона с параметрами $\alpha \in(0,2]$, $\theta \in[-1,1]$.

Для симметричньх $\xi$ выполняется $\gamma=1 / 2$,

$$
\mathbf{P}\left(S_{n} \leqslant 0\right)-\frac{1}{2}=\frac{1}{2} \mathbf{P}\left(S_{n}=0\right)<\frac{c}{\sqrt{n}},
$$

так что ряд (6) сходится, $G(z)=\exp \left\{-\frac{1}{2} \sum z^{n} \mathbf{P}\left(S_{n}=0\right) / n\right\}$.

Из сказанного видно, что в отличие от случая $A_{-}$, здесь влияние хвостов распределения $\xi$ на асимптотику (2) в [1] менее значимо.

Существует обширная литература о скорости сходимости $F^{(n)}(t)=\mathbf{P}\left(S_{n} / \sigma_{n} \leqslant t\right)$ при соответствующей нормировке $\sigma_{n} \mathrm{~K} F_{\alpha, \theta}(t)$. В ней можно найти, в частности, условия, достаточные для сходимости ряда (6) в случае $\mathrm{E} \xi^{2}=\infty$. Мы укажем здесь в качестве иллюстрации лишь один из известных нам результатов.

Пусть $\nu_{r}=\int \mid x^{r}\left(P(d x)-d F_{\alpha, \theta}(x) \mid<\infty\right.$ npu $r>\alpha u \int x\left(P(d x)-d F_{\alpha, \theta}(x)\right)=0$ в случае $\alpha \geqslant 1$. Тогда

$$
\sup _{t}\left|F^{(n)}(t)-F_{\alpha, \theta}(t)\right| \leqslant c \nu_{r} n^{1-r / \alpha}
$$

(см. [3], [4]).

Очевидно, что в этом случае сходимость (6) будет иметь место.

Получим теперь одно уточнение теоремы 8 , касающееся локальны $x$ теорем для $\eta_{-}$. В дальнейшем соотношение $g_{n} \sim c f_{n}$ при $c=0$ мы будем понимать как $g_{n}=o\left(f_{n}\right)$.

Теорема 9. 1) Пусть выполнено $A_{0} u$

$$
\Delta_{n} \sim c n^{-\gamma}, \quad 0 \leqslant c<\infty, \quad \gamma>0
$$

при $n \rightarrow \infty$. Тогда

$$
\mathbf{P}\left(\eta_{-}=n\right) \sim \frac{\gamma n^{-\gamma-1}}{\Gamma(1-\gamma)} G(1), \quad 0<G(1)<\infty .
$$

2) Если $\mathrm{E} \xi=0, \mathrm{E}|\xi|^{3}<\infty$ и распределение $\xi$ либо решетчато, либо

$$
\limsup _{|t| \rightarrow \infty}|\varphi(i t)|<1
$$

то $\gamma=1 / 2$ и выполнено (7), (8).

Аналогичные соотношения справедливы для $\mathbf{P}\left(\eta_{+}=n\right)$. 
ДокАЗАТЕЛЬСТво. В силу (18) в [1]

$$
\mathrm{E} z^{\eta_{-}}=1-\exp \left\{-\gamma \sum_{k=1}^{\infty} \frac{z^{k}}{k}-\sum \frac{z^{k} \Delta_{k}}{k}\right\}=1-(1-z)^{\gamma} G(z)
$$

Асимптотика коэффициентов $a_{k}$ разложения функции

$$
a(z)=-(1-z)^{\gamma}=\sum_{k=0}^{\infty} a_{k} z^{k}
$$

хорошо известна:

$$
a_{n} \sim \frac{\gamma n^{-1-\gamma}}{\Gamma(1-\gamma)}
$$

Для выяснения асимптотики коэффициентов $g_{k}$ разложения

$$
G(z)=\sum_{k=0}^{\infty} g_{k} z^{k}
$$

нам понадобится вспомогательное утверждение.

Пусть $d_{n} \sim c n^{-\alpha}, \alpha>1,0 \leqslant c<\infty$,

$$
d(z)=\sum_{k=0}^{\infty} d_{k} z^{k}, \quad \hat{d}_{n}=\left|d_{n}\right|, \quad \hat{d}(z)=\sum_{k=0} \hat{d}_{k} z^{k}
$$

Пусть далее $\mathscr{A}(\lambda)$ аналитическая функция в области $|\lambda| \leqslant \hat{d}(1)$, так что

$$
\mathscr{A}(\lambda)=\sum_{k=0}^{\infty} A_{k} \lambda^{k}, \quad\left|A_{k}\right| \leqslant c_{1}(\hat{d}(1)(1+\varepsilon))^{-k}
$$

при некоторых $\varepsilon>0, c_{1}<\infty$. Так как ряд $d(z)$ абсолютно сходится, по теореме Винера-Леви функция $\mathscr{A}(d(z))$ также представима в виде абсолютно сходящегося ряда:

$$
\mathscr{A}(d(z))=\sum_{k=0}^{\infty} g_{k} z^{k}
$$

ЛЕмма 1. При выполнении названных выше условий

$$
g_{n} \sim \mathscr{A}^{\prime}(d(1)) d_{n}
$$


Это утверждение переносит известные результаты об асимптотике субэкспоненциальных хвостов (см. [5]) на локальные асимптотические свойства регулярных знакопеременных последовательностей. Доказательство леммы см. в [6].

Воспользуемся леммой 1 при $d_{n}=-\Delta_{n} / n, \mathscr{A}(\lambda)=e^{\lambda}$. Тогда $G(z)=\mathscr{A}(d(z))$ и $(7)$ будет означать вьполнение условий леммы при $\alpha=1+\gamma$. Из (9), (10) получаем

$$
\mathbf{P}\left(\eta_{-}=n\right)=\sum_{k=0}^{n} a_{k} g_{n-k}=\sum_{k \leqslant n / 2}+\sum_{k>n / 2}
$$

где

$$
\sum_{k \leqslant n / 2}=g_{n} a(1)+o\left(g_{n}\right)=o\left(g_{n}\right), \quad \sum_{k>n / 2}=a_{n} G(1)+o\left(a_{n}\right) .
$$

Это доказьвает (8).

Второе утверждение теоремы следует из того, что в условиях п. 2 теоремы

$$
\Delta_{n}=\mathbf{P}\left(S_{n} \leqslant 0\right)-\frac{1}{2}=\frac{c \mathrm{E} \xi^{3}}{\sqrt{n}}+o\left(\frac{1}{\sqrt{n}}\right)
$$

(см., например, [7]) и, стало быть, выполнено (7) при $\gamma=1 / 2$.

Теорема доказана.

2.2. Фиксированный уровень $x>0$. Асимптотика распределений $\eta_{ \pm}(x)$ при $x>0$ отличается от соответствующей асимптотики для $\eta_{ \pm}$множителем, зависящим лишь от $x$. Именно, в [2] и [8]-[11] установлено, что в условиях теорем 2 (для класса $\mathscr{R}), 5,6$ в [1] и теоремы 8 справедлива

Теорема 10. В случаях $A_{-}, A_{0}$ при $n \rightarrow \infty$ июбом фиксированном $x \geqslant 0$

$$
\frac{\mathbf{P}\left(\eta_{-}(x)>n\right)}{\mathbf{P}\left(\eta_{-}>n\right)} \rightarrow r_{-}(x), \quad \frac{\mathbf{P}\left(n<\eta_{+}(x)<\infty\right)}{\mathbf{P}\left(n<\eta_{+}<\infty\right)} \rightarrow r_{+}(x),
$$

где функиии $r_{ \pm}(x)$ найдень в явном виде.

Вид функций $r_{ \pm}(x)$ оказьвается весьма сложным. В следующем разделе будет найдена асимптотика этих функций при $x \rightarrow \infty$.

Для ограниченных решетчатых $\xi$ в [12] получены асимптотические разложения для $\mathbf{P}\left(\eta_{ \pm}(x)=n\right)$.

\section{3. Растущий уровень $x$}

В этом разделе нам будет удобнее изменить направление сноса в том случае, когда он не нуль, так что теперь основным случаем будет $A_{+}=\{a=\mathrm{E} \xi>0\}$, а основным объектом изучения - время $\eta_{+}(x)$ первого прохождения уровня $x \rightarrow \infty$. В связи с этим класс $\mathscr{R}$ будет описываться теперь с помощю регулярных левы $x$ хвостов

$$
\left\{W(t)=\mathbf{P}(\xi<-t)=t^{-\beta} L_{W}(t)\right\},
$$


где $\beta>1, L_{W}-\mathrm{MM} \Phi$ при $t \rightarrow \infty$.

Мы будем рассматривать в этом разделе лиш классы хвостов $\mathscr{R}$ и $\mathscr{C}$, а также распределения из класса $\mathscr{M}_{s}$, для которых $\mathrm{E}|\xi|^{s}<\infty$. Ясно, что пересечение классов $\mathscr{M}_{s}$ и $\mathscr{R}$ не пусто. Так как $\left\{\eta_{+}(x)>n\right\}=\left\{\bar{S}_{n} \leqslant x\right\}$, нашу задачу можно рассматривать так же, как задачу о “малых уклонениях" максимума $\bar{S}_{n}$.

3.1. Класс хвостов $\mathscr{C}$. В этом случае асимптотика $\mathbf{P}\left(\eta_{+}(x)>n\right)=\mathbf{P}\left(\bar{S}_{n} \leqslant x\right)$ изучена достаточно полно в [13], [14] при любом значении $a=\mathrm{E} \xi$. Метод исследования, использованньй в [13], [14], состоит в отыскании двойных преобразований над $\mathbf{P}\left(\eta_{+}(x)>n\right.$ ) (по $x$ и по $n$ ) в терминах решений уравнений типа Винера-Хопфа (или, что то же - в терминах компонент факторизации; в [13] эти компоненты находятся в явном виде) и в последующем асимптотическом обращении этих преобразований. Сравнительно недавно выяснилось, что такой подход не является единственно возможньм в рассматриваемых задачах. В [15], [16] прямьми вероятностньми методами было установлено, что асимптотики вероятностей больших (и малых) уклонений $\bar{S}_{n}$ в ряде случаев могут быть явньм образом найдены через соответствующую асимптотику для $S_{n}$. В частности, если $x / n<a=\mathrm{E} \xi, x / n \in\left(\alpha_{1}, \alpha_{2}\right)$, где $\alpha_{i}$ определяются аналитическими свойствами $\varphi(\lambda)$, то

$$
\mathbf{P}\left(\eta_{+}(x)>n\right) \sim c\left(\frac{x}{n}\right) \mathbf{P}\left(S_{n} \leqslant x\right),
$$

где $c(v)$ найдена в явном виде. Более подробно на случае $\mathscr{C}$ мы здесь останавливаться не будем.

3.2. Класс распределений $\mathscr{M}_{s}$. Для распределений из класса $\mathscr{M}_{s}$ без привлечения свойств регулярности (12) удается изучить асимптотику $\mathbf{P}\left(\eta_{+}(x)>n\right)$ лишш в случае $A_{0}=\{a=0\}$.

Везде в дальнейшем будет предполагаться, что в решетчатом случае уровень $x \rightarrow \infty$ принадлежит соответствующей решетке.

Теорема 11. Пусть выполнено $\mathscr{M}_{3}, a=0, \mathrm{E} \xi^{2}=1, x \rightarrow \infty, x=o(\sqrt{n})$. Тогда

$$
\mathbf{P}\left(\eta_{+}(x)>n\right) \sim \frac{x \sqrt{2}}{\sqrt{\pi n}} .
$$

Это утверждение немедленно следует из оценки скорости сходимости

$$
\sup _{x}\left|\mathbf{P}\left(\bar{S}_{n} \leqslant x\right)-2\left[\Phi\left(\frac{x}{\sqrt{n}}\right)-\frac{1}{2}\right]\right|<\frac{c}{\sqrt{n}}, \quad c<\infty
$$

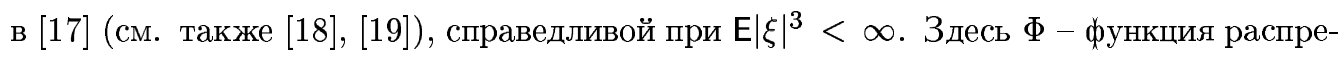
деления стандартного нормального закона. Так как $\Phi(u)-1 / 2 \sim u / 2 \pi$ при $u \rightarrow 0$, мы получаем (14).

Если распределение $\xi$ решетчато или $\lim \sup _{t \rightarrow \infty} \varphi(i \lambda)<1$, то при выполнении $\mathscr{M}_{s}$, $s>3$, можно изучать также асимптотическое разложение для $\mathbf{P}\left(\bar{S}_{n} \leqslant x\right)$ при $x \rightarrow \infty$ (см. [18], [14], [20], [21]) и можно получать аналогичные разложения и для

$$
\mathbf{P}\left(\eta_{+}(x)=n\right) \sim \frac{x}{\sqrt{2 \pi} n^{3 / 2}}
$$


(локальные предельные теоремы для $\eta_{+}(x)$ ).

Случай $x \rightarrow \infty, a>0, \mathscr{M}_{s}$, насколько нам известно, остается не изученным. Изучению этого случая в классе $\mathscr{R}$ и посвящен следующий раздел.

3.3. Асимптотика $\mathbf{P}\left(\eta_{+}(x)>n\right)$ при выполнении условий $x \rightarrow \infty, a>0, \mathscr{R}$. Наряду с названными в заголовке этого раздела условиями нам может понадобиться в случае $\beta \in(1,2)$ (см. (12)) дополнительное условие

$$
\mathbf{P}(\xi>t) \leqslant V(t)
$$

где $V$ имеет вид $V(t)=t^{-\alpha} L(t), \alpha>1, L-\mathrm{MM}$. Обозначим

$$
z=a n-x .
$$

Теорема 12. Пусть $a>0, x \rightarrow \infty, x<$ an и выполнено $\mathscr{R}$. Тогда

1) $Е с л и \mathrm{E} \xi^{2}<\infty(\beta>2) u z \gg \sqrt{n \ln n}, m o$

$$
\mathbf{P}\left(\eta_{+}(x)>n\right) \sim \frac{x}{a} W(z)
$$

2) соотношение (17) сохранится, если $\beta \in(1,2)$, выполнено (16), n и z такоsbl, чmo

$$
n W(z) \rightarrow 0, \quad n V\left(\frac{z}{\ln z}\right) \rightarrow 0 .
$$

Смысл утверждения (17) весьма прост: основной вклад в вероятность $\mathbf{P}\left(\bar{S}_{n} \leqslant x\right)$ вносят траектории, у которых на одном из первых $x / a$ скачков (до пересечения границы $x$ "линией сноса" $\left.a(k)=\mathrm{E} S_{k}=a k\right)$ произошел отрицательньй выброс на величину $\leqslant x-$ an.

Из теоремы следует

$$
\mathbf{P}\left(\bar{S}_{n} \leqslant x\right) \sim \frac{x}{a n} \mathbf{P}\left(S_{n} \leqslant x\right),
$$

так что соотношение (13) здесь сохраняется, но вместо весьма сложного множителя $c(\alpha)$ в $(13)$, здесь стоит значительно более простая функция $c(\alpha)=\alpha / a$, при этом случай $\alpha \rightarrow 0$ не исключается.

ДокАЗАТЕЛЬСтво теоремы 12 разобьем на три этапа.

1. Оценка снизу. Обозначим

$$
G_{n}=\left\{\bar{S}_{n} \leqslant x\right\}, \quad B_{j}=\left\{\xi_{j}<-z(1+\varepsilon)\right\}, \quad v=\frac{x}{a}(1-\varepsilon)
$$

где $\varepsilon>0$ - фиксированное малое число. Тогда, считая для простоты $v$ целочисленным, будем иметь

$$
\mathbf{P}\left(G_{n}\right) \geqslant \mathbf{P}\left(G_{n} \cup_{j=1}^{v} B_{j}\right)=\sum_{j=1}^{v} \mathbf{P}\left(G_{n} B_{j}\right)+O\left((x W(z))^{2}\right) .
$$


Очевидно, что при $j \leqslant v$ в силу закона больших чисел $\mathbf{P}\left(G_{n} / B_{j}\right) \rightarrow 1$ при $x \rightarrow \infty$. Так как $x W(z) \rightarrow 0$, то

$$
\mathbf{P}\left(G_{n}\right) \geqslant \sum_{j=1}^{v} \mathbf{P}\left(B_{j}\right)(1+o(1))+O\left((x W(z))^{2}\right) \sim \frac{x}{a}(1-\varepsilon) W(z(1+\varepsilon)) .
$$

В силу произвольности $\varepsilon>0$ находим окончательно

$$
\mathbf{P}\left(G_{n}\right) \geqslant \frac{x}{a} W(z)(1+o(1))
$$

2. Оценка $\mathbf{P}\left(G_{n}\right)$ сверху для срезанных слагаемых. Обозначим $C_{j}=\left\{\xi_{j}>-z / r\right\}$, $r>1, C=\bigcap_{j=1}^{n} C_{j}$. Тогда

$$
\mathbf{P}\left(G_{n}\right)=\mathbf{P}\left(G_{n} C\right)+\mathbf{P}\left(G_{n} \bar{C}\right)
$$

где $\bar{C}$ есть дополнение к $C$,

$$
\mathbf{P}\left(G_{n} \bar{C}\right) \leqslant \sum_{j=1}^{n} \mathbf{P}\left(G_{n} \bar{C}_{j}\right), \quad \mathbf{P}\left(G_{n} C\right) \leqslant \mathbf{P}\left(S_{n}-a n \leqslant-z ; C\right) .
$$

ЛЕмма 2. Если $\mathrm{E} \xi^{2}<\infty,(\beta>2), u x \gg \sqrt{n \ln n}, m o$

$$
\mathbf{P}\left(G_{n} C\right) \leqslant(n W(z))^{r+o(1)} .
$$

Если $\beta \in(1,2)$, выполнено (16), (18), то

$$
\mathbf{P}\left(G_{n} C\right) \leqslant c(n W(z))^{r} .
$$

Утверждение леммы вытекает из теоремы 4.1 (следствие 4.1) и теоремы 3.1 в [22]. Если выбрать $r>\beta /(1-\beta)$, то, очевидно

$$
(n W(z))^{r}=o(x W(z)), \quad \mathbf{P}\left(G_{n} C\right)=o(x W(z)) .
$$

3. Оценка сверху суммы (21). Имеем

$$
\mathbf{P}\left(G_{n} \bar{C}_{j}\right)=\mathbf{P}\left(\bar{S}_{j-1} \leqslant x, \xi_{j}<-\frac{z}{r}, S_{j-1}+\xi_{j}+\bar{S}_{n-j}^{*} \leqslant x\right),
$$

где $\bar{S}_{n-j}^{*}$ имеют распределение $\bar{S}_{n-j}$, но не зависят от $\xi_{1}, \ldots, \xi_{j}$. Так как $\xi_{j}$ не зависит от $\xi_{1}, \ldots, \xi_{j-1}$ и $\bar{S}_{n-j}^{*}$, то

$$
\begin{aligned}
\mathbf{P}\left(G_{n} \bar{C}_{j}\right) & \leqslant \mathrm{E}\left[W\left(-x+\bar{S}_{n-j}^{*}+S_{j-1}\right) ; \bar{S}_{n-j}^{*}+S_{j-1}>x+\frac{z}{r}\right] \\
& \leqslant \mathrm{E} W\left(\max \left(\frac{z}{r},-x+S_{n-1}\right)\right)=\mathrm{E} W\left(\max \left(\frac{z}{r}, z+S_{n-1}^{0}\right)\right) \\
& \sim W(z) \mathrm{E}\left[\max \left(\frac{1}{r}, 1+\frac{S_{n}^{0}}{z}\right)\right]^{-\beta},
\end{aligned}
$$


где $S_{n}^{0}=S_{n}-a n$. Так как $S_{n}^{0} / z \underset{p}{\rightarrow} 0$ при выполнении условий $z \gg \sqrt{n \ln n}$ или $(18)$, мы получаем отсюда

$$
\mathbf{P}\left(G_{n} \bar{C}_{j}\right) \leqslant W(z)(1+o(1)) .
$$

Кроме того,

$$
\mathbf{P}\left(G_{n} \bar{C}_{j}\right) \leqslant W\left(\frac{z}{r}\right) \mathbf{P}\left(\bar{S}_{j-1} \leqslant x\right)=W\left(\frac{z}{r}\right) \mathbf{P}(\eta(x) \geqslant j) .
$$

Поэтому

$$
\sum_{j=1}^{n} \mathbf{P}\left(G_{n} \bar{C}_{j}\right) \leqslant \frac{x}{a}(1+\varepsilon) W(z)+W\left(\frac{z}{r}\right) \sum_{j>\frac{x}{a}(1+\varepsilon)} \mathbf{P}(\eta(x) \geqslant j) .
$$

Здесь $W\left(\frac{z}{r}\right) \leqslant c W(z)$ и в силу усиленного закона больших чисел и теоремы восстановления $\mathrm{E} \eta_{+}(x)=\sum \mathbf{P}\left(\eta_{+}(x)>j\right) \sim x / a$ находим, что второе слагаемоев правой части $(23)$ есть $o(x) W(z)$. Отсюда следует, что

$$
\sum_{j=1}^{n} \mathbf{P}\left(G_{n} \bar{C}_{j}\right) \leqslant \frac{x}{a} W(z)(1+o(1))
$$

Сопоставляя это неравенство с (20)- $(22)$, мы получим такую же оценку для $\mathbf{P}\left(G_{n}\right)$. Это вместе с оценкой снизу (19) доказывает теорему.

\section{СПИСОК ЦИТИРОВАННОЙ ЛИТЕРАТУРЫ}

[1] Боровков А. А. Об асимптотике распределений времен первого прохождения. I // Матем. заметки. 2004. Т. 75. №1. С. 24-39.

[2] Bingham N. H., Goldie C. M., Teugels J. L. Regular Variation. Cambridge: Cambridge University Press, 1987.

[3] Паулаускас В. И. Оценки остаточного члена в предельной теореме в случае устойчивого предельного закона // Литовский матем. сб. 1974. Т. 14. № 1. С. 165-187.

[4] Паулаускас В.И. Равномерные и неравномерные оценки остаточного члена в предельной теореме в случае устойчивого закона // Литовский матем. сб. 1974. Т. 14. №4. С. 171-185.

[5] Chover J., Ney P., Wainger S. Function of probability measures // J. Analyse. Math. 1973. V. 26. P. 255-302.

[6] Боровков А.А. О субэкспоненциальных распределениях и асимптотике распределения максимума последовательных сумм // Сиб. матем. ж. В печати.

[7] Петров В. В. Суммы независимых случайных величин. М.: Наука, 1971.

[8] Emery D. J. Limiting behaviour of the distribution of the maxima of partial sums of certain random walks // J. Appl. Probab. 1972. V. 9. P. 572-579.

[9] Doney R.A. On the asymptotic behaviour of first passage times for transient random walk // Probability Theory and Related Fields. 1989. V. 18. P. 239-246.

[10] Bertoin J., Doney R. A. Some asymptotic results for transient random walks // Adv. Appl. Probab. 1996. V. 28. P. 207-227.

[11] Могульский А. А., Рогозин Б. А. Случайные блуждания в положительном квадранте I-III // Матем. труды. 1999. Т. 2. № 2. С. 57-97; 2000. Т. 3. №1. С. 48-118; 2001. Т. 4. № 1. C. 1-25.

[12] Боровков А. А. Вероятностные процессы в теории массового обслуживания. М.: Наука, 1972. 
[13] Боровков А. А. Предельные теоремы о распределении максимума сумм ограниченньх решетчатых случайных величин. I, II // Теория вероятн. и еепримен. 1960. Т. 5. № 2. С. 137-171; I960. T. 5. № 4. C. 377-392.

[14] Боровков А. А. Новые предельные теоремы в граничных задачах для сумм независимых слагаемых // Сиб. матем. ж. 1962. Т. 3. № 5. С. 645-694.

[15] Боровков А. А. О преобразовании Крамера, больших уклонениях в граничных задачах и условном принципе инвариантности // Сиб. матем. ж. 1995. Т. 36. № 3. С. 493-509.

[16] Боровков А. А. Об условных распределениях, связанных с большими уклонениями // Сиб. матем. ж. 1996. Т. 37. № 4. С. 732-744.

[17] Нагаев С. В. О скорости сходимости распределения максимума сумм независимых случайных величин // Теория вероятн. и ее примен. 1970. Т. 15. № 2. С. 320-325.

[18] Нагаев С. В. Асимптотические разложения для максимума сумм независимых случайных величин // Теория вероятн. и ее примен. 1970. Т. 15. № 3. С. 527-528.

[19] Нагаев С. В. О скорости сходимости в одной граничной задаче // Теория вероятн. и ее примен. 1970. Т. 15. № 2. С. 179-199.

[20] Королюк В.С. К асимптотике распределений максимальных уклонений // Докл. АН CCCP. 1962. T. 142. № 3. C. 522-525.

[21] Королюк В.С. Асимптотический анализ распределений максимальных уклонений в решетчатой схеме блужданий // Теория вероятн. и ее примен. 1962. Т. 7. № 4. С. 393-409.

[22] Боровков А. А. Оценки для распределения сумм и максимумов сумм случайных величин при невыполнении условия Крамера // Сиб. матем. ж. 2000. Т. 5. № 5. С. 997-1038.

Институт математики им. С. Л. Соболева СО РАН

Поступило

E-mail: borovkov@math.nsc.ru

17.05.2002

Исправленный вариант

01.04 .2003 